\title{
Gamified Crowdsourcing for Disaster Risk Management
}

\author{
Antonella Frisiello, Quynh Nhu Nguyen, Claudio Rossi, Fabrizio Dominici \\ Istituto Superiore Mario Boella \\ via Pier Carlo Boggio 61, 10138, Torino, Italy \\ Email: \{surname\}@ismb.it
}

\begin{abstract}
Nowadays, the number and the magnitude of natural hazards are increasing. During emergency situations different forms of cooperation take place, including the crowdsourcing, which is envisioned by many Disaster Risk Management approaches to enable citizens to support the emergency management process. However, crowdsourcing is a challenging paradigm as it requires a sustained engagement in order to be effective. In this paper we propose a gamification strategy for crowdsourced Disaster Risk Management services aimed to increase awareness, engagement, and change people behaviors.
\end{abstract}

Keywords-Gamification; Crowdsourcing; Disaster Risk Management; Citizen science.

\section{INTRODUCTION}

Nowadays we observe extreme weather and serious environmental events all around the world: everyone can be potentially affected by natural hazards such as floods, fires, extreme weather events. The exposure to such hazards cause different reactions, both at a collective and individual level, including spontaneous and volunteer citizens' activities. During an emergency, different forms of cooperation take place, including the so called digital activism. The volunteer contribution of people to an ad-hoc activity [1], which typically consists in producing, collecting, sharing information on specific topics, is known as crowdsourcing. In crisis situations, it has been observed that crowdsourcing is successful in increasing participation and produced contents [2], [3]. Because its potential benefits, the most recent Disaster Risk Management (DRM) approaches include the direct participation of citizens along the different stages [4], [5], [6]. Crowdsourcing can support activities aiming at preventing and reducing disaster risks. It can work as a tool for enabling different forms of information exchange between citizens and professional responders. Crisis managers can crowdsource hazards monitoring activities to: track the current state of the affected areas, perform damage assessments, localize affected people and resources on the ground. Nevertheless, the connection between the crowd and DRM professionals is not easy, due to the extremely different nature of the involved subjects. From one side, the emergency organizations are hierarchical, while on the other side, the crowd is non-hierarchical and self-organized by definition [7]. This is also valid for digital environments. For instance, social media would be and effective and pow- erful crowdsourcing platforms, but data collection, fusion, and processing of heterogeneous unstructured data across different platforms, as well as issues related to ethics and privacy, make their integration into DRM a very challenging task. Ad-hoc crowdsourcing platforms can mitigate some of these obstacles, since they typically allow to collect more structured data [8], [9], which are closer to requirements of emergency organizations. Crowdsourcing leverages on the willingness of people to voluntarily and freely contribute to a common goal. However, the precondition to succeed is a consistent participation of people [10], [11]. In particular, the amount of crowd-sourcers impacts both on the quantity and the quality of collected data, as well as on geographic coverage. The key factor to reach an high level of participation is sustained people engagement. An approach progressively more applied to create and foster people engagement is gamification [12]. According to literature, games might foster civic engagement and active citizenship to crowdsource real-world problems [13], [14]. Gamification works in this direction, applying game thinking to non-gameful contexts [15]. In comparison to traditional games, gamification aims to increase awareness, engagement, and people behaviors change. Both gamification and crowdsourcing are techno-social paradigms that leverage on voluntary participation.

This paper presents the design of a gamification strategy for crowdsourcing DRM applications encompassing data collection and validation. The rest of this paper is organized as follows. In Section II we review related works, while in Section III we explain the gamification approach together with its main methodological frameworks. In Section IV we describe the design of the gamification strategy for crowdsourcing data collection and validation tasks in DRM. Finally, in Section $\mathrm{V}$ we present the conclusions and the envisaged future works.

\section{RELATED WORKS}

A number of crowdsourcing platforms and tools have played a role in DRM. Among the most relevant examples we find Ushahidi ${ }^{1}$, a platform originally created for monitoring the Kenya election, and later extended to build maps from reports gathered by voluntary groups during

\footnotetext{
${ }^{1}$ www.ushahidi.com (Accessed on Sept. 2017)
} 
natural disasters. Another one is Mobile4D ${ }^{2}$, which is a mobile crowdsourcing disaster alerting and reporting system, enabling affected people to directly report disasters and use personal communication channels to coordinate action and advices [16]. Initially, gamification approaches were investigated as social experiments. For example, DARPA ${ }^{3}$ launched in 2009 the Red Balloon Challenge, which asked people to locate for a money prize ten red weather balloons secretly distributed. The challenge was solved in around eight hours and more than 350k people participated [17]. This experiment showed how much the crowdsourcing can be efficient in performing a given task, spotting objects, and collecting information. Further DARPA's experiments allowed to better focus on the use of economic rewords as motivational driver. Crowdsourcing systems are increasingly implementing gamification elements [18] in several domains, including environmental monitoring. In this domain, the majority of works explore platforms that implements entertaining ways to learn, increasing awareness and hopefully change citizens attitudes towards self-protection behaviors [19], [20], [21], [22]. Among these, very few projects specifically address natural hazards [23], [24], [25]. FLOODIS ${ }^{4}$ allows citizens and responders to send geolocalized flood reports containing a picture along with the perceived water level through their mobile devices, and use the gathered data to improve flood mapping, nowcasting, and forecasting. Similarly, iSeeChange ${ }^{5}$ uses crowdsourcing for collecting users reports on environmental phenomena such as unusual weather patterns. In this case, users can also search existing reports by area, date and investigation type; and they can also update already submitted reports. The engagement strategy in this case is based on the dissemination of the generated contents, which can be shared and voted by users within the community, producing quantitative indicators that are used for ranking contents. The socialization is the strategic key also for SharkBase ${ }^{6}$, a platform addressing young people that want to become "citizen scientists". The main goal of the service is to map the distribution of shark populations around the world. The participation is reinforced through a public leaderboard and by peer-to-peer validation. All these examples put in evidence the application of some gamification components. Nevertheless, gamifying is something more complex.

\section{Gamify to EnGAGE}

The word gamification has come to the attention of the world in $2010^{7}$. Recently it has become a trending topic in a number of fields related to user engagement, service

\footnotetext{
${ }^{2}$ www.mobile4d.capacitylab.org (Accessed on Sept. 2017)

${ }^{3}$ The U.S. Defense Advanced Research Projects Agency.

${ }^{4}$ www.floodis.eu (Accessed on Sept. 2017)

${ }^{5}$ www.iseechange.org (Accessed on Sept. 2017)

${ }^{6}$ www.shark-base.org (Accessed on Sept. 2017)

${ }^{7}$ Year in which the Merriam-Webster vocabulary records the word Gamification
}

design, marketing [26] and also in the academia, which has been producing more studies in this subject. According to the most quoted definition, gamification refers to the application of game thinking in non-game contexts, with the aim to motivate desired behaviors [27]. The core principle is that playing, as innate human activity, contains elements that work as native cues that can be potentially applicable to any domain.In comparison to traditional games, which are only aimed to create enjoyment, gamification incorporates game's elements for goals related to real environments [28]. Gamification relies on game design and profits from scientific grounds and empirical approaches, as reviewed in [29]. In the majority of published frameworks, design and implementation processes are User-Centric and leverage on user research and iterative evaluation sessions. The user perspective is considered fundamental, and translated into a cognitive model that maps motivations and goals to game mechanisms in order to engage the target. Such frameworks define the core elements to be addressed by the gamification design. Motivators, mechanics, dynamics, narrative and aesthetic, as well as rewards have to be combined into a flowing experience. One of the most known and applied operative gamification approach is the Mechanics Dynamic Aesthetics (MDA) [30] that refers to three building blocks valid for the whole process: during the design, the development and the experience. The first block refers to Mechanics, which includes all the game components at the data representation level, including algorithms. Dynamics describe the system behavior resulting from the players inputs on mechanics, while Aesthetics refer to the emotional responses we want to evoke during the experience. The core structure of gamification grounds on endogenous values to be made explicit in order to better engage players [31]. Human activities are driven by motivations, which are the core psychological aspects to be taken into account when designing a gamified experience. In comparison to external ones, inner motivations result more effective to engage people, both in normal conditions as well during the emergency response phase, when the motivation to act is expected to be stronger than in other phases [7]. Nevertheless, from a DRM perspective, the willingness of people to cooperate is very important also in the prevention and the preparedness phases, when crowdsourcing monitoring activities may results particularly helpful. One of the most used and effective motivational frameworks applied in game design is the socalled RAMP [32], an acronym that refers to the four main intrinsic levers that move players: (1) the relatedness, referring to the need of being connected with other people; aspects such as identity, socialization, membership, and sharing reply to this need; (2) the autonomy, referring to the need of expressing creativity, and experiment while being in control; (3) the mastery, related to the need to know, learn, and become even more keen on some topic or ability; (4) the purpose, which concerns the meaningfulness 
of actions we are doing. The RAMP is a simple model that can be effectively adopted in the early stages of game design to shape challenges, incentives, rewards, events, aesthetics and all the fundamental design bricks of the gamification strategy.

\section{Gamification as a Strategy to Facilitate the INTEGRATION OF CROWDSOURCING IN DRM}

We propose a novel gamification strategy aimed at fostering the key tasks of a crowdsourcing applications in DRM, focusing on data collection and validation tasks. Such strategy is aimed to strengthen the citizen engagement toward topics related to cooperative environmental monitoring, natural hazards, risk awareness. The challenge is to design a mix of tools and resources for citizens able to provide reliable data for emergency responders and decision makers. In order to achieve this objectives, three specific goals have been set and entrusted to gamification:

- Citizens' awareness: raising the peoples interest and attention on environmental risks and informing them about natural hazards is of paramount importance to obtain valuable and actionable data;

- Citizens' engagement in reporting: the in-field data collection (reporting) is the key added value that crowdsourcing can provide to DRM because it can achieve distributed monitoring, delivering both high quantity and high accuracy of information;

- Reports validation: the data collection process requires a validation mechanisms because citizens-generated information can be inaccurate, inappropriate, or counterfeit.

The aforementioned goals directly map into the core functions we propose to gamify, designing challenges and actions for crowdsourcers (players). We design our gamification strategy following the MDA approach, which translates in the following operational steps:

1) Outline the context and the target users in a systemic view;

2) Identify motivators and map them as Competences required to effectively contribute to the crowdsourcing objective;

3) Define for each competence a list of traceable Activities (quests) to be performed by players;

4) Define the Score system, and the rules to assign points for performed activities;

5) Define the Reward system, articulated in Achievements, to be progressively gained, and Awards, which are periodically assigned according to performance indicators;

6) Define the Level System that is used to assign a given status to the player according to its points and Achievements.

7) Outline the recognition systems and the Aesthetic guidelines for a general crowdsourcing application for

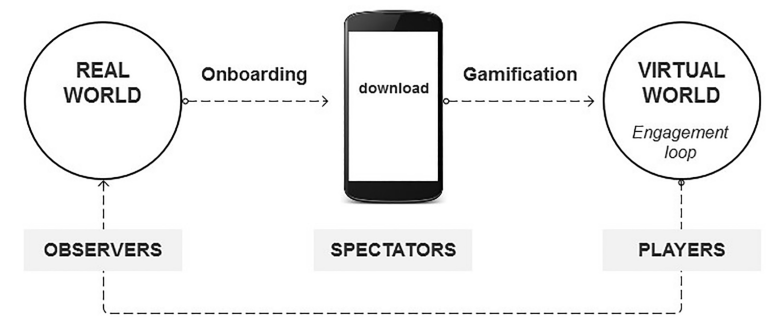

Figure 1: Actors in the gamified crowdsourcing scenario.

\section{DRM.}

\section{A. The Target Audience}

The first step to gamify an experience is to identify the actors to be reached and involved, both directly and indirectly. According to [33], the actors are distinguished according to the involvement extent, the interaction style (active vs. passive) and the experience (absorption vs. immersion). More in detail, a gamified ecosystem has to take in account the following types of actors:

1) Players: the active performers that compete in the experience and are highly immersed;

2) Spectators: individuals who are part of the real environment (e.g. audience, supervisors, etc.), not directly competing, and whose presence influence how the gamified experience works;

3) Observers: external individuals who are passively involved in the experience. They have no direct impact on the gamified experience but they are able to watch it from the outside. Their presence and amount are can affect the popularity of the experience, and they can also be considered as potential players or spectators.

The aforementioned user categorization (Figure 1) can be easily and effectively declined to the DRM context. Players are the operational profiles involved as crowdsourcers, i.e., citizens willing and able to cooperate.Spectators are decision makers working in control rooms (e.g., fire services, public authorities, civil protections, technical services, meteorological agencies). They will receive and benefit from the crowdsourced information. Despite they do not directly compete, they can enter into the gamified process opening calls for reports (that in gamification terms means creating quests for players) and validating received reports. Finally, observers refer to the rest of the population, media, outsiders, as well as distant witnesses, who can be potentially involved.

\section{B. Competences}

Within the DRM context, the main goals are (i) raising awareness on natural hazard, (ii) stimulate protective behaviors toward both the environment and people, (iii) enable an effective cooperation with emergency organizations. In 
our strategy we map such goals to Competences, which are progressively achieved by performing a set of associated Actions (quests), leading players into their journey throughout the Level System. The Competences we identify are:

1) Socializer: this competence has been designed to grow the player community, which is a necessary condition for the crowdsourcing. It responds to the relatedness need and entails very simple actions that does not require domain expertise, i.e., inviting friends to join the community and sharing information. For this reason, we enable such actions at all levels.

2) Learner: users are invited to build up their knowledge on environment and natural hazards both in a "passive" way (e.g., by reading tips), as well in "active" mode (e.g., by answering to quizzes). From the crowdsourcing perspective this phase is relevant to introduce and train users to produce good quality data.

3) Reporter: this competence allows users to provide data from the field, and it is at the core of the crowdsourcing system. Also in this case, we foresee two modes to stimulate users, who are allowed both to spontaneously send reports, as well as to answer to specific request from the system (created by Spectators). We refer to the latter case as the provision of on-demand reports.

4) Reviewer: in order to be included in the DRM and used by emergency managers, reports have to be accurate. For this, reason, Reviews is the highest Competence with respect to the level system. Users that have been good reporters can become reviewers and peer-review reports done by other players by upvoting (confirm) or downvoting (reject) them. On top of this level, there is only the Authority validation, which is a necessary step to consider the information fully reliable for DRM professionals.

A graphical representation of the aforementioned competences, which are inspired to the RAMP model, is shown in Figure 2 together with their associated activities. The activities clustered by Competence can be progressively enabled to engages users in a growth path, which starts with the Learner, and ends with the Reviewer. The latter is enable upon gaining the Reported role, which is the core of the crowdsourcing.

\section{Score and Reward systems}

In gamification, points are the granular units of measurement through which the system keeps count of player's actions. A wide range of points can be assigned and combined, as suggested by [34]. The Score system has to assign points coherently with the importance of goals and activities. In the context of DRM, the Reporters' activities are the most valuable and complex, thus they should award the greatest amount of points. We give a greater amount of points to ondemand reports against spontaneous one because the former are requested by DRM managers, hence more relevant for

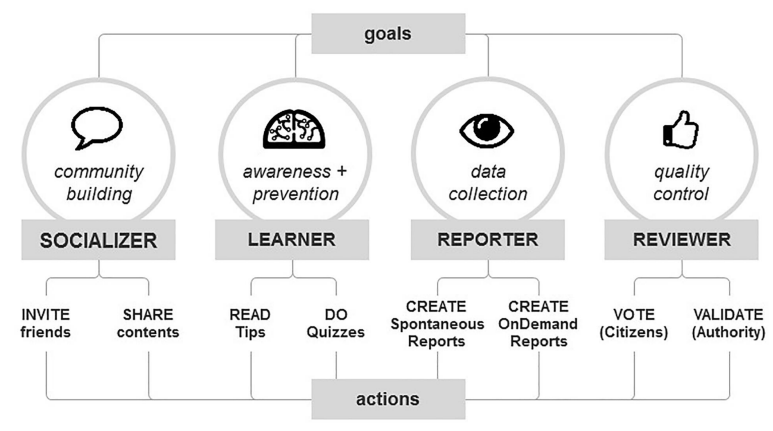

Figure 2: Goals, Competences and Users Activities of the proposed gamification strategy.

them. Tasks assigned to Learners are also important, because they prepare the user for reporting activity, and they provide the initial positive feedbacks required to keep the user in the flow. Reviewers' tasks allow to gain points, but their rate has to be a bit lower because this activity can be performed quickly and on a potentially large number of reports. We made Reporters to gain or lose points according to the validation done by the authority. If a report is validated, the Reporter gains an additional $50 \%$ of the points initially awarded for the report creation. Conversely, if a report gets rejected we distinguish between two cases: (i) the report is rejected because not appropriate, meaning that it is completely out of scope (e.g., explicit contents, an image of a water bottle is sent along with the indication of flood, etc.); (ii) the report is inaccurate (e.g., the image is blurred and/or badly cropped with respect to the relevant content). Inaccurate reports results into a loss of $50 \%$ of the points initially awarded for the report being rejected, while not appropriate reports cause a loss of $150 \%$. This means that in the latter case the Reporter will lose points from the total score (i.e., half of the points initially awarded for the report), while in the former case the user will retain half of the points initially awarded. This mechanism should encourage inaccurate reporters to improve in the task, and strongly discourage the generation of not appropriate contents. At the same time, also Reviewers' score gets affected by the validation from the authority so as to promote good reviewers. Specifically, a Reviewed gets a gain or loss of $100 \%$ if the report is validated in accordance to the review (i.e., validated when upvoted or rejected when downvoted) or in discordance (i.e., rejected when upvoted or validated when downvoted), respectively. Socializers tasks produce the least amounts of points, but the actions do not require any specific domain knowledge and they are so simple that it is easy for users to improve the personal score inviting friends and sharing contents. In addition, the Score system includes a dummy level, namely the Starter, that is meant to welcome a new user and invite him/her to participate. Also in this case simple actions are important to onboard people (e.g., do the onboarding, login, and trainings on how to report and 
review).

Points gained performing the gamified actions allow users to gain two types of rewards. Achievements, which provide stable status, are related to both Competences and domainspecific challenges, and assessed on the base of quantitative criteria. Achievements provide players visibility and incremental statuses, and they are generally visible in a "locked" status until the players meet the criteria to "unlock" them. Symbols and graphical representation are very effective to visualize them. A typical example is represented by Medals - with a progression that is mapped into precious metals (i.e., bronze, silver, gold, platinum) and associated to titles that explains the achievement reached (e.g., 'Bronze Learner', 'Golden Reviewers, etc.). Note that this is an arbitrary choice that can be changed according to the Aesthetic strategy implemented. Conversely, Awards are periodically assigned bonus, which are valid within a given time frame, and similarly to Achievements they refer to a specific challenges. Awards are used to reward the most active users with additional points and temporary statuses publicly visible to the user community. We suggest to create special badges for each key activity and further differentiate them according to Hazards. Badges can be earned completing a full set of hazard-specific activities, providing evidence of the user's specialization (e.g., 'Fire Reporter'). In the Reward system, Competences and domain elements are intertwined and work together to focus and engage players on the critical activities of the crowdsourcing.

From a strategical perspective, Achievements are used also to define barriers and controls the players progression through the Level system. Such barriers ensures that users develops the right combination of competences along the game.

The summary of all considered actions, the associated achievements, and the awards is reported in Table I. Note that only recurrent activities can be linked to Awards, which are periodic. Hence, there is no Awards for reading tips and doing quizzes because the content available impose a certain limit, after which the activity is no longer possible. We do not specify exactly the points that should be awarded for each action, the thresholds to be used to define achievements, and the points awarded for gaining achievements and awards, because all these elements depend upon the specific application, the context, the available content, the Level System (Subsection IV-D), and the target longevity of the game.

\section{The Level System}

The activities each user does into the system are translated into points. This results in a total Player Score, which is corresponds to a Status inside the Level System. The Level system should be carefully designed to propose an initially easy and progressively challenging path able to enhance the accountability of citizens, transforming them from passive spectators into active and relevant part of a DRM processes.

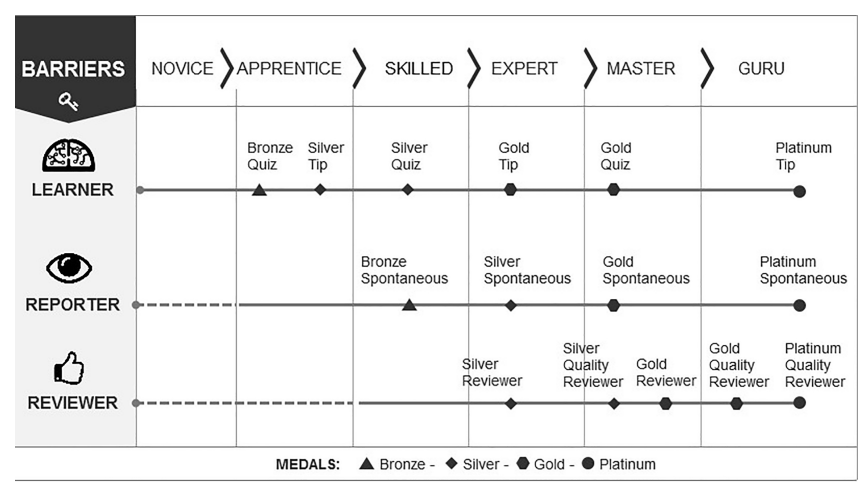

Figure 3: Mapping between statuses, Competence, and barriers (Achievements).

The Level system is one of the highest components of motivation for gamers, since it clearly displays the current status of the player and which are the possible progressions available. In the DRM context we propose a sixstep Level System having the following statuses: Novice, Apprentice, Skilled, Experts, Master, Guru. Such statuses are general and can be applied in any situation. In order to become more engaging, the statuses naming could be customized according to the DRM context and even to the specific application according to the Aesthetic implemented (Subsection IV-F). Discounting the actions included in the Socializer competence, which do not require a specific domain knowledge, we map statuses to Competences, according to the skills required to perform them. Assuming that a new player is not familiar with the DRM context, we let a Novice to be a Learner, while we enable Reporter and Reviewer activities starting from the Apprentice and the Skilled level, respectively. The rationale behind these choices is that a player must have some knowledge in DRM (achieved through learning) before to start reporting, and that the reporting activity should be well experienced before starting to review reports from other players. Furthermore, we use Achievements as barriers after the Apprentice level so as to force players to progressively familiarize with unlocked activities before progressing to the next level. Note that the longevity of the game strongly depends on the threshold assigned to each level in terms of Total Score and on the Tuning of such barriers. The realization of the aforementioned strategy is shown in Figure 3.

\section{E. Leaderboards}

Leaderboards are a powerful game mechanic to engage users, since they provide status recognition within the community and a synthetic feedback on the provided contribution. A leaderboard displays a ranking of players, highlighting the best performance in terms of Scores. Nevertheless, depending on what they show, leaderboards can be demotivating: a top-10 list would not easily host newbies or medium contributors in a huge community of users, making them feel frustrated. To prevent these feelings, [34] suggests 
Table I: Actions together with associated Achievement and Awards of the proposed gamification strategy.

\begin{tabular}{|c|c|c|c|c|}
\hline \multirow{2}{*}{ Competence } & \multirow{2}{*}{ Actions } & \multicolumn{2}{|c|}{ Achievements } & \multirow{2}{*}{ Awards } \\
\hline & & Medal & Hazard Badges & \\
\hline \multirow{2}{*}{ Socializer } & Share content on personal social network & Sharer & $\mathrm{v}$ & $\mathrm{v}$ \\
\hline & Invite friends to download the app & Promoter & - & $\mathrm{v}$ \\
\hline \multirow{2}{*}{ Learner } & Read TIP & Reader & $\mathrm{v}$ & - \\
\hline & Answer QUIZ & Learner & $\mathrm{v}$ & - \\
\hline \multirow{6}{*}{ Reporter } & Do training report & & - & - \\
\hline & Spontaneous Report & Spontaneous Reporter & $\mathrm{v}$ & $\mathrm{v}$ \\
\hline & On-demand Report & Reporter on-demand & $\mathrm{v}$ & $\mathrm{v}$ \\
\hline & Report get validated from authority & - & - & - \\
\hline & Report get rejected as not appropriate by authority & - & - & - \\
\hline & Report get rejected as not accurate by authority & - & - & - \\
\hline \multirow{4}{*}{ Reviewer } & Do training on review & - & - & - \\
\hline & Vote a report (up or down) & Reviewer & - & $\mathrm{v}$ \\
\hline & Authority validate a report I have upvoted, or reject a report I have downvoted & Quality Reviewer & - & $\mathrm{v}$ \\
\hline & Authority validate a report I have downvoted, or reject a report I have upvoted & - & - & - \\
\hline \multirow{2}{*}{ General } & Login & - & - & - \\
\hline & Do onboard (ex. Tour of main functionalities of app) & - & - & - \\
\hline
\end{tabular}

to display contextual leaderboards that place the player in the middle of the list, among other players (above and below) with similar scores. Leaderboards play a role in the experience flow, both motivating players to compete with closest co-players and defending the position while climbing up. Considering the specific domain of DRM, we propose to include geographically based leaderboards because natural hazards and risks are strongly related to the territory. This allows players to select a reference area on a range, scaling from the city level up to regional, national level.

\section{F. Aesthetics}

In game design, Aesthetics describe the desirable emotional responses we want to evoke in players when they interact with a game system [30]. Elements such as visual narrative and metaphors are able to raise emotion and require a careful design able to connect the experience with the real context of reference. All the elements of the gamification strategy - Competences, Activities, the Reward system, Medals, Badges and Awards, Statuses - require to be designed to match the Aesthetic with the context in order to provide sense of coherence, positive emotions and meaning. A human-centric design allows to connect game elements, working as extrinsic motivators, with the intrinsic users motivations. In addition, to successfully gamifying a crowdsourcing system, a narrative has to provide a sense of relevance to both individuals and the community, while fostering their sense of social responsibility, which in turn motivates them to continue participating. Since the Aesthetic is strongly depended on the specific application, we avoid the definition of a generalized aesthetic framework for crowdsourcing applications in DRM, as it may not be the best solution.

\section{COnclusions And Future Works}

Crowdsourcing enables active citizenship through community and informal participation, enabling to collectively solve big challenges. It can be leveraged to improve DRM processes by allowing citizens to contribute in monitoring and reporting activities, which are of paramount important across the full cycle of the emergency management. In this context, we have designed a gamification strategy as an engagement tool aimed at fostering the crucial activities of data collection and validation. Our strategy reviews and applies the main ingredients proposed by the current literature, and declines them in the DRM domain. We believe that a gamified approach can be promising to involve and engage more people in DRM processes, stimulating an active attitude and self-protection behaviors. Furthermore, it can sustain data collection for monitoring natural hazards through the crowdsourcing, which can ultimately reduce impacts in terms of human and economic losses in case of natural disasters.

Future works include the implementation of a gamified mobile application for crowdsourcing DRM tasks, and its evaluation in terms of user experience, engagement, and quality of the contents produced.

\section{ACKNOWLEDGMENT}

This work was partially funded by the European Commission through the I-REACT project (H2020-DRS-1-2015), grant agreement n.700256.

\section{REFERENCES}

[1] J. Howe, Crowdsourcing: Why the Power of the Crowd Is Driving the Future of Business. Crown Publishing Group, 2008.

[2] P. Meier, "Crisis mapping in action: How open source software and global volunteer networks are changing the world, one map at a time," Journal of Map \& Geography Libraries, vol. 8, no. 2, pp. 89-100, 2012.

[3] A. Olteanu, S. Vieweg, and C. Castillo, "What to expect when the unexpected happens: Social media communications across crises," in Conference on Computer Supported Cooperative Work; Social Computing. ACM, 2015, pp. 994-1009.

[4] C. Aydin, C. Tarhan, A. Ozgur, and V. Tecim, "Improving disaster resilience using mobile based disaster management system," Procedia Technology, vol. 22, pp. 382-390, 2016. 
[5] G. Foresti, M. Farinosi, and M. Vernier, "Situational awareness in smart environments: socio-mobile and sensor data fusion for emergency response to disasters," Journal of Ambient Intelligence and Humanized Computing, vol. 6, no. 2, pp. 239-257, 2015.

[6] C. Rossi, M. H. Heyi, and F. Scullino, "A service oriented cloudbased architecture for mobile geolocated emergency services," Concurrency and Computation: Practice and Experience, vol. 29, no. 11, pp. e4051-n/a, 2017.

[7] G. Schimak, D. Havlik, and J. Pielorz, "Crowdsourcing in crisis and disaster management - challenges and considerations." in Environmental Software Systems. Infrastructures, Services and Applications. ISESS 2015, ser. IFIP Advances in Information and Communication Technology, R. D. et. al, Ed., vol. 448. Springer, 2015, pp. 56-70.

[8] J. de Albuquerque, M. Eckle, B. Herfort, and A. Zipf, Crowdsourcing geographic information for disaster management and improving urban resilience: an overview of recent developments and lessons learned. Ubiquity Press, 2016, ch. 23, pp. 309-321.

[9] C. Rossi, W. Stemberger, C. Bielski, G. Zeug, N. Costa, D. Poletto, E. Spaltro, and F. Dominici, "Coupling crowdsourcing, earth observations, and e-gnss in a novel flood emergency service in the cloud," in International Geoscience and Remote Sensing Symposium (IGARSS), 2015, pp. 2703-2706.

[10] C. Park and E. Johnston, "Crowdsourced, voluntary collective action in disasters," in Annual International Conference on Digital Government Research. ACM, 2015, pp. 329-330.

[11] J. McGonigal, Reality Is Broken: Why Games Make Us Better and How They Can Change the World. Penguin Group, 2011.

[12] A. Darejeh and S. Salim, "Gamification solutions to enhance software user engagement-a systematic review," International Journal of HumanComputer Interaction, vol. 32, no. 8, pp. 613-642, 2016.

[13] C. Raphael, C. Bachen, K. Lynn, J. Baldwin-Philippi, and K. McKee, "Games for civic learning: A conceptual framework and agenda for research and design," Games and Culture, vol. 5, no. 2, pp. 199-235, 2010.

[14] H. Jenkins, "Confronting the challenges of participatory culture: Media education for the 21 st century," 2006

[15] S. Deterding, D. Dixon, R. Khaled, and L. Nacke, "From game design elements to gamefulness: Defining gamification," in Proceedings of the 15th International Academic MindTrek Conference: Envisioning Future Media Environments, ser. MindTrek '11. ACM, 2011, pp. 9-15.

[16] L. Frommberger and F. Schmid, "Mobile4d: Crowdsourced disaster alerting and reporting," in Proceedings of the Sixth International Conference on Information and Communications Technologies and Development, vol. 2. ACM, 2013, pp. 29-32.

[17] J. Tang, M. Cebrian, N. Giacobe, H. Kim, T. Kim, and D. Wickert, "Reflecting on the darpa red balloon challenge," Commun. ACM, vol. 54, no. 4, pp. 78-85, 2011.

[18] K. Seaborn and D. Fels, "Gamification in theory and action," Int. J. Hum.-Comput. Stud., vol. 74, no. C, pp. 14-31, 2015.
[19] J. Lee, P. Ceyhan, W. Jordan-Cooley, and W. Sung, "Greenify: a real-world action game for climate change education," Simulation \& Gaming, vol. 44, no. 2-3, pp. 349-365, 2013.

[20] F. R., F. P., and C. Pasini, SnowWatch: A Multi-modal Citizen Science Application. Springer International Publishing, 2016, pp. 538-541.

[21] M. A.D., G. Michalakidis, and P. Krause, "Tiger nation: Empowering citizen scientists," in 6th IEEE International Conference on Digital Ecosystems and Technologies (DEST), 2012, pp. 1-5.

[22] N. Prestopnik and J. Tang, "Points, stories, worlds, and diegesis," Comput. Hum. Behav., vol. 52, no. C, pp. 492-506, 2015.

[23] R. Netek and J. Panek, "Framework see-think-do as a tool for crowdsourcing support - case study on crisis management," in ISPRS - International Archives of the Photogrammetry, Remote Sensing and Spatial Information Sciences, vol. XLI-B6, 2016, pp. 13-16.

[24] E. McCartney, K. Craun, E. Korris, D. Brostuen, and L. Moore, "Crowdsourcing the national map," Cartography and Geographic Information Science, vol. 42, pp. 54-57, 2015.

[25] N. Moreno, S. Savage, A. Leal, J. Cornick, M. Turk, and T. Hllerer, "Motivating crowds to volunteer neighborhood data," in Conference Companion on Computer Supported Cooperative Work; Social Computing. ACM, 2015, pp. 235-238.

[26] J. Hamari, J. Koivisto, and H. Sarsa, "Does gamification work? a literature review of empirical studies on gamification," in Hawaii International Conference on System Sciences. IEEE, 2014, pp. 30253034.

[27] S. Deterding, "Gamification: Designing for motivation," interactions, vol. 19 , no. 4, pp. 14-17, 2012

[28] A. Marczewski, "Gamification design vs game design," 2014, online; accessed 26/09/2017. [Online]. Available: www.gamified.uk/2014/ 03/25/gamification-design-vs-game-design

[29] A. Mora, D. Riera, C. Gonzalez, and J. Arnedo-Moreno, "A literature review of gamification design frameworks," in International Conference on Games and Virtual Worlds for Serious Applications (VS-Games), 2015, pp. 1-8.

[30] R. Hunicke, M. Leblanc, and R. Zubek, "Mda: A formal approach to game design and game research," in In Proceedings of the Challenges in Games AI Workshop, Nineteenth National Conference of Artificial Intelligence, 2004, pp. 1-5.

[31] J. Schell, The Art of Game Design: A Book of Lenses. Morgan Kaufmann, 2008.

[32] A. Marczewski, "Understanding intrinsic motivation with ramp," 2013, online; accessed 26/09/2017. [Online]. Available: http://www.gamification.co/2013/05/01/ understanding-intrinsic-motivation-with-ramp/

[33] K. Robson, K. Plangger, J. Kietzmann, I. McCarthy, and L. Pitt, "Is it all a game? understanding the principles of gamification," Business Horizons, vol. 58, no. 4, pp. 411-420, 2015.

[34] G. Zichermann and C. Cunningham, Gamification by Design: Implementing Game Mechanics in Web and Mobile Apps. O'Reilly Media, 2011. 